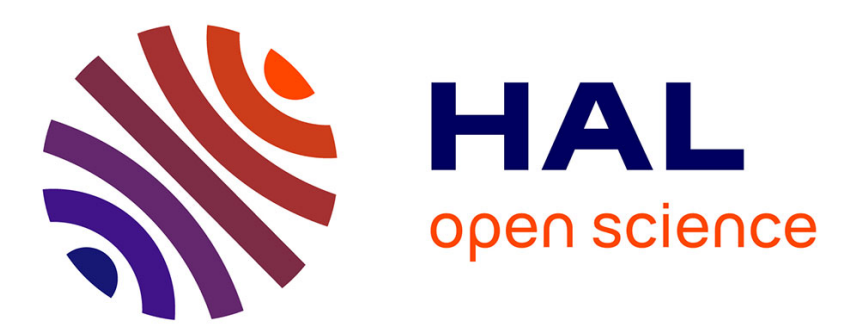

\title{
On boundedness of solutions of state periodic systems: a multivariable cell structure approach
}

Denis Efimov, Johannes Schiffer

\section{To cite this version:}

Denis Efimov, Johannes Schiffer. On boundedness of solutions of state periodic systems: a multivariable cell structure approach. IEEE Transactions on Automatic Control, 2019, 64, 10, pp.4094-4104. 10.1109/TAC.2019.2892395 . hal-01966708

\section{HAL Id: hal-01966708 \\ https://hal.inria.fr/hal-01966708}

Submitted on 29 Dec 2018

HAL is a multi-disciplinary open access archive for the deposit and dissemination of scientific research documents, whether they are published or not. The documents may come from teaching and research institutions in France or abroad, or from public or private research centers.
L'archive ouverte pluridisciplinaire HAL, est destinée au dépôt et à la diffusion de documents scientifiques de niveau recherche, publiés ou non, émanant des établissements d'enseignement et de recherche français ou étrangers, des laboratoires publics ou privés. 


\title{
On boundedness of solutions of state periodic systems: a multivariable cell structure approach
}

\author{
Denis Efimov, Johannes Schiffer
}

\begin{abstract}
Many dynamical systems are periodic with respect to several state variables. This periodicity typically leads to the coexistence of multiple invariant solutions (equilibria or limit cycles). As a consequence, while there are many classical techniques for analysis of boundedness and stability of such systems, most of these only permit to establish local properties. Motivated by this, a new sufficient criterion for global boundedness of solutions of such a class of nonlinear systems is presented. The proposed method is inspired by the cell structure approach developed by Leonov and Noldus and characterized by two main advances. First, the conventional cell structure framework is extended to the case of dynamics, which are periodic with respect to multiple states. Second, by introducing the notion of a Leonov function the usual definiteness requirements of standard Lyapunov functions are relaxed to sign-indefinite functions. Furthermore, it is shown that under (mild) additional conditions the existence of a Leonov function also ensures input-to-state stability (ISS), i.e., robustness with respect to exogenous perturbations. The performance of the proposed approach is demonstrated via the global analysis of boundedness of trajectories for a nonlinear system.
\end{abstract}

\section{INTRODUCTION}

In control systems and many related domains, such as mechanical, transportation, power and biological systems, stability is one of the most fundamental and sought-after properties of a dynamical system [8], [10], [24], [26], [27], [28], [33], [39]. Until today, most available approaches for stability analysis of nonlinear systems are based on Lyapunov theory [33]. A key feature of a Lyapunov-based stability analysis is that boundedness and convergence properties of the system's solutions can be assessed without explicit computation of the latter. Instead, it suffices to verify a set of inequalities for the Lyapunov function and its time derivative, which is derived with respect to the system's equations. More precisely, the existence of a continuously differentiable (or at least Lipschitz continuous) Lyapunov function, which is positive definite with respect to an equilibrium (or an invariant set) and the time derivative of which is non-positive along the solutions of the system under investigation, is equivalent to stability of that equilibrium (or set). Similarly, instability of an equilibrium can be studied using the Chetaev function approach [10], [16].

The preliminary formulations of the main results of this paper are given in [17] without proofs

D. Efimov is with Inria, Univ. Lille, CNRS, UMR 9189 - CRIStAL, F59000 Lille, France and with Department of Control Systems and Informatics, University ITMO, 49 avenue Kronverkskiy, 197101 Saint Petersburg, Russia. Email: denis.efimov@inria.fr.

J. Schiffer is with Control Systems and Network Control Technology, Brandenburg University of Technology (BTU), 03046, Cottbus, Germany. Email: schiffer@b-tu.de.

This work was supported in part by the Government of Russian Federation (Grant 08-08) and the Ministry of Education and Science of Russian Federation (Project 14.Z50.31.0031).
A Chetaev function may be sign-indefinite ${ }^{1}$ with a negative or positive definite derivative.

The classical stability theory in the sense of Lyapunov has mainly focused on properties of a single isolated equilibrium point. Yet, in many (engineering) applications, such as biological and power systems or distributed optimization, the systems under study possess several equilibria or invariant sets (including hidden attractors [14]). The aim for a rigorous treatment of such systems has motivated the extension of Lyapunov-based stability theory to dynamical systems with several disjoint invariant sets [4], [34], [24], [38], [6], [44], [20], [15]. This extension is far from trivial and requires suitable modifications and relaxations of the employed stability notions, see, e.g., [15] and the subsequent developments in [2], [3] for an appropriate extension of the input-to-state stability (ISS) property in the presence of multiple equilibria. Further related results on robust stability analysis of systems with multiple equilibria are [1], [5], [9].

A special case of systems often exhibiting multiple equilibria is represented by dynamics, which is periodic with respect to one or several of its states. Paramount examples of such systems are the forced nonlinear pendulum [19], [21], power systems [36], [40], [12], [46] and microgrids [42], [11], phase-locked loops [29], [30] and complex networks of oscillators [44], [13], [45]. Yet unfortunately, the application of the aforementioned existing results to periodic systems is in most cases very challenging. The main reason for this is the technical difficulty of constructing suitable Lyapunov functions. For example, if some of the states of the system are periodic (e.g., they evolve on the circle), in order to be able to apply the approach proposed in [3] the corresponding Lyapunov function also has to be periodic with respect to these states, which is a severe requirement. As a consequence, available results are often limited to local stability claims, see [13].

Motivated by this wide range of potential applications, we consider a special class of systems, which possess periodic right-hand sides with respect to several state components and the state of which evolves in $\mathbb{R}^{n}$. For such systems, the two main contributions of the present paper are: to derive sufficient conditions for global boundedness of trajectories via the concept of a Leonov function and to show that under (mild) additional conditions the existence of a Leonov function readily implies input-to-state stability (ISS), i.e., robustness with respect to exogenous perturbations. We remark that in the context of systems with infinitely many equilibria, boun-

\footnotetext{
${ }^{1}$ A function $V: \mathbb{R}^{n} \rightarrow \mathbb{R}$ is called sign-definite if $V(0)=0$ and $V(x)>$ 0 for all $x \in \mathbb{R} \backslash\{0\}$ or $V(x)<0$ for all $x \in \mathbb{R} \backslash\{0\}$; and it is called sign-indefinite if $V(x)$ takes both, positive and negative, values.
} 
dedness of trajectories is the natural counterpart to stability of a single equilibrium point in the classical Lyapunov theory.

With regard to boundedness of solutions, our approach is inspired by the cell structure framework proposed in [31] (and later in [35]) and further developed in [24], [48] for systems, the dynamics of which are periodic with respect to a scalar state variable. The following observation lies at the core of the cell structure approach of Leonov and collaborators. At first, exploiting the periodicity of the dynamics allows to construct - via a function, which is sign-indefinite in the periodic state - unbounded forward invariant sets, which in addition are globally attractive. Second, the intersection of these unbounded forward invariant sets consists of globally attractive compact forward invariant and isolated sets, i.e., cells, which gives the name to this method.

The restriction to periodicity of the dynamics with respect to a scalar state variable is essential to obtain forward invariant sets in [31], [35], since in the scalar case the individual cells are only connected via equilibria. But, clearly, this restriction is a severe limitation in the analysis of complex networks with periodic dynamics. Therefore, in the present paper we extend the approach of [31], [35] to systems the dynamics of which are periodic with respect to multiple state variables by proposing a multivariable cell structure approach.

As noted in [35], such extension is far from trivial. In fact, while as done in [31], [35], we also exploit the periodicity of the dynamics to construct (unbounded) forward invariant sets, in the multivariable setting significant additional care has to be taken to obtain compact forward invariant sets, i.e., cells. This technical difficulty arises from the fact that in the multivariable case, the cells can, in general, not only be connected via equilibria. This challenge is overcome in the present paper by introducing the concept of a Leonov function. Loosely speaking, a Leonov function is sign-indefinite with respect to the periodic states and radially unbounded with respect to the non-periodic states. Furthermore, a Leonov function is negative definite with respect to the distance to a set, which separates the equilibria of the system. This last requirement is a major difference compared to the functions employed in [31], [35] and is key to establish our main result.

Besides boundedness of solutions, robustness with respect to exogenous disturbances is often a highly desirable property in many control engineering applications. Therefore, a second contribution of the paper is to provide additional conditions under which the existence of a Leonov function also implies the ISS property. This claim is established by leveraging recent results in [18], where an extension of the ISS theory from [3] to periodic systems has been proposed. Furthermore, the extension in [18] has been derived for systems on manifolds and, thus, does not allow to establish boundedness of trajectories in $\mathbb{R}^{n}$, which is studied in this work. Furthermore, the presented results are tested via application to a complex nonlinear system.

We anticipate that the proposed multivariable cell structure approach may prove useful in a variety of scenarios and applications. For instance, the original cell structure approach has recently been combined in [7] with LaSalle's invariance principle and in [43] with the ISS result of [18] to establish global properties of a synchronous machine infinite bus system. The extensions of this analysis to the multi-machine case [41] and to the Kuramoto model by using the methods derived in the present paper are under current investigation. Furthermore, the existence of a compact forward invariant set is a prerequisite for many methods that allow to assess the asymptotic behavior of a system's trajectories. Such methods comprise the classical LaSalle invariance principle [47], but also contraction [22], [32], respectively convergence [37], or differential positivity [23].

The remainder of this paper is structured as follows. Preliminaries and the theory from [24], [48] are given in Section II. The problem statement is presented in Section III with the main results in Section IV. Robustness with respect to external inputs is investigated in Section V. The efficacy of the presented approach is illustrated in Section VI.

\section{PRELIMINARIES}

Denote the sets of real and integer numbers by $\mathbb{R}$ and $\mathbb{Z}$, respectively, and let $\mathbb{R}_{+}=\{s \in \mathbb{R}: s \geq 0\}, \mathbb{Z}_{+}=\mathbb{Z} \cap \mathbb{R}_{+}$.

Let the map $f(x): \mathbb{R}^{n} \rightarrow \mathbb{R}^{n}$ be of class $\mathcal{C}^{1}, f(0)=0$, and consider a nonlinear system of the following form:

$$
\dot{x}(t)=f(x(t)), \quad \forall t \geq 0
$$

with state $x(t) \in \mathbb{R}^{n}$. We denote by $X(t, x)$ the uniquely defined solution of (1) at time $t$ fulfilling $X(0, x)=x$. A set $S \subset M$ is invariant for the system (1) if $X(t, x) \in S$ for all $t \in \mathbb{R}$ and for all $x \in S$; for $x \in \mathbb{R}^{n}$ the point $y \in \mathbb{R}^{n}$ belongs to its $\omega$-limit ( $\alpha$-limit) set if there is a sequence $t_{i}, \lim _{i \rightarrow+\infty} t_{i}=+\infty$, such that $\lim _{i \rightarrow+\infty} X\left(t_{i}, x\right)=y$ $\left(\lim _{i \rightarrow+\infty} X\left(-t_{i}, x ; 0\right)=y\right)$; for any $x \in \mathbb{R}^{n}$ its $\alpha$ - and $\omega$ limit sets are invariant [25]. We define the distance from a point $x \in \mathbb{R}^{n}$ to the set $S \subset \mathbb{R}^{n}$ as $|x|_{S}=\inf _{a \in S}|x-a|$, where $|x|=|x|_{\{0\}}$ for $x \in \mathbb{R}^{n}$ is a usual Euclidean norm of a vector $x \in \mathbb{R}^{n}$. Furthermore, we introduce the vector norm

$$
|x|_{\infty}=\max _{1 \leq i \leq n}\left|x_{i}\right|
$$

then

$$
|x|_{\infty} \leq|x| \leq \sqrt{n}|x|_{\infty}
$$

A continuous function $\alpha: \mathbb{R}_{+} \rightarrow \mathbb{R}_{+}$belongs to class $\mathcal{K}$ if $\alpha(0)=0$ and the function is strictly increasing. The function $\alpha: \mathbb{R}_{+} \rightarrow \mathbb{R}_{+}$belongs to the class $\mathcal{K}_{\infty}$ if $\alpha \in \mathcal{K}$ and its supremum is infinity. A continuous function $\beta: \mathbb{R}_{+} \times \mathbb{R}_{+} \rightarrow$ $\mathbb{R}_{+}$belongs to the class $\mathcal{K} \mathcal{L}$ if $\beta(\cdot, t) \in \mathcal{K}_{\infty}$ for each fixed $t \in \mathbb{R}_{+}$and $\lim _{t \rightarrow+\infty} \beta(s, t)=0$ for each fixed $s \in \mathbb{R}_{+}$.

In this work we assume the following:

Assumption 1. Let $x=(z, \theta) \in \mathbb{R}^{n}$, where $z \in \mathbb{R}^{k}$ and $\theta \in \mathbb{R}^{q}$ are two subsets of the state vector, $n=k+q, k>0$ and $q>0$. The vector field $f$ in (1) is $2 \pi$-periodic with respect to $\theta$.

In other words, we suppose that the system (1) can be embedded into a manifold $M=\mathbb{R}^{k} \times \mathbb{S}^{q}$ by a simple projection of the variables $\theta$ on the sphere $\mathbb{S}^{q}$. 


\section{A. Boundedness of solutions of periodic systems}

As outlined in Section I, the present paper is rooted on the stability analysis of periodic systems given in [24], [48], where Assumption 1 is satisfied for $q=1$. Next, we recall the sufficient criterion derived in [31], [24], [48], which allows to establish boundedness of solutions of periodic systems with a scalar periodic state. To this end consider a special case of the system (1) given by

$$
f(x)=P x+b \varphi\left(c^{\top} x\right),
$$

where $P \in \mathbb{R}^{n \times n}$ is a singular matrix, $c \in \mathbb{R}^{n}, b \in \mathbb{R}^{n}$, $\varphi: \mathbb{R} \rightarrow \mathbb{R}$ is a $\Delta$-periodical Lipschitz continuous function, $\varphi(0)=0$. We note that a time-varying and discontinuous version of $\varphi$ has been considered in [24], [48], but - to simplify the exposition - we restrict ourselves to the autonomous and continuous version of $\varphi$. Then under these restrictions and for any initial condition $x_{0} \in \mathbb{R}^{n}$ the system (1) has a solution $X\left(t, x_{0}\right)$. Assume also that for all $\sigma \in \mathbb{R} \backslash\{0\}$

$$
\mu_{1} \leq \frac{\varphi(\sigma)}{\sigma} \leq \mu_{2}
$$

for some $\mu_{1}<0$ and $\mu_{2}>0$.

Theorem 1. [31], [24], [48] Assume that there exists $\lambda>0$ such that:

1) the matrix $P+\lambda I_{n}$, where $I_{n} \in \mathbb{R}^{n \times n}$ is the identity matrix, has $n-1$ eigenvalues with negative real parts;

2) for all $\omega \in \mathbb{R}$

$$
\mu_{1}^{-1} \mu_{2}^{-1}+\left(\mu_{1}^{-1}+\mu_{2}^{-1}\right) \operatorname{Re} \chi(i \omega-\lambda)+|\chi(i \omega-\lambda)|^{2} \leq 0,
$$

where $\chi(s)=c^{T}\left(P-s I_{n}\right)^{-1} b$.

Then, for any initial condition $x_{0} \in \mathbb{R}^{n}$ the solution $X\left(t, x_{0}\right)$ of the periodic system (1) is bounded for $t \in$ $[0,+\infty)$.

The proof of Theorem 1 (see Theorem 4.3.1 in [24], or Theorem 4.7 in [48]) is based on the fact that under the introduced conditions there is $H=H^{\top} \in \mathbb{R}^{n \times n}$ (which has one negative and $n-1$ positive eigenvalues) such that for $V_{0}(x)=x^{\top} H x$ we have that $d V_{0}(x(t)) / d t \leq-2 \lambda V_{0}(x(t))$ for all $t \in[0,+\infty)$, which implies that the set $\Omega_{0}=$ $\left\{x \in \mathbb{R}^{n}: V_{0}(x) \leq 0\right\}$ is forward invariant for (1), i.e. $X\left(t, x_{0}\right) \in \Omega_{0}$ for all $t \in[0,+\infty)$ provided that $x_{0} \in \Omega_{0}$. Next, introducing the functions $V_{j}(x)=V_{0}(x-j \delta)$ and sets $\Omega_{j}=\left\{x \in \mathbb{R}^{n}: V_{j}(x) \leq 0\right\}$, where $j$ is any integer and the vector $\delta \in \mathbb{R}^{n}$ satisfies the conditions $\delta \neq 0, P \delta=0$ and $c^{\top} \delta=\Delta$, by periodicity of $f$ in (1) we obtain that $d V_{j}(x(t)) / d t \leq-2 \lambda V_{j}(x(t))$ for all $t \in[0,+\infty)$, then the sets $\Omega_{j}$ are forward invariant for (1). Finally, it is shown in [24], [48] that for any $x_{0} \in \mathbb{R}^{n}$ there is an index $j_{0}$ such that $x_{0} \in \Gamma_{j_{0}}$, where $\Gamma_{j}=\Omega_{j} \cap \Omega_{-j} \cap\left\{x \in \mathbb{R}^{n}:\left|h^{\top} x\right| \leq j\left|h^{\top} \delta\right|\right\}$ with $h \in \mathbb{R}^{n}$ being the eigenvector of the matrix $H$ corresponding to the negative eigenvalue. As it has been shown above $X\left(t, x_{0}\right) \in \Gamma_{j_{0}}$ for all $t \in[0,+\infty)$ (since it is true for $\Omega_{j_{0}} \cap \Omega_{-j_{0}}$ ). In addition the set $\Gamma_{j_{0}}$ is bounded, which was necessary to prove. In other words, an important observation of [31], [24], [48] is that any intersection of the sets $\Omega_{j}$ for all integers $j$ forms a kind of cell cover of $\mathbb{R}^{n}$, where each cell is bounded and forward invariant. Therefore, this framework is commonly known as cell structure approach.

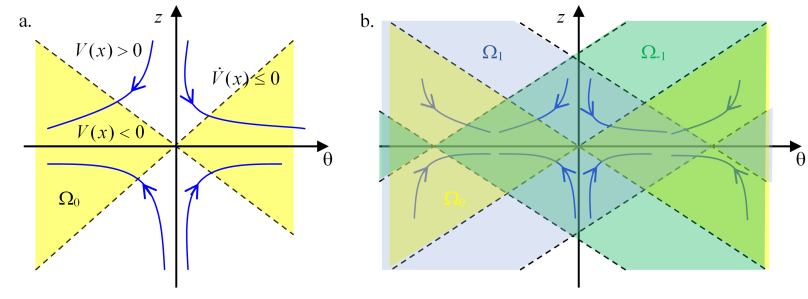

Figure 1. Illustration of the sets $\Omega_{j}$ for $j \in\{-1,0,1\}$

\section{B. An illustrative example}

The following example illustrates the main idea of the proof of Theorem 1.

Example 1. Suppose (1) is given by $x=(z, \theta) \in \mathbb{R}^{2}$ and $f: \mathbb{R}^{2} \rightarrow \mathbb{R}^{2}$. Furthermore, suppose $f$ is periodic in $\theta$ with period $T>0$, i.e., for any integer $j \in \mathbb{Z}$, we have that $f(z, \theta+$ $T j)=f(z, \theta)$. Following Theorem 1 , suppose that there exists a function $V: \mathbb{R}^{2} \rightarrow \mathbb{R}$, such that for some $\lambda>0$,

$$
\begin{gathered}
V(0,0)=0, \quad V(z, 0)>0, \quad \forall z \in \mathbb{R} \backslash\{0\}, \\
\frac{\partial V(x)}{\partial x} f(x) \leq-\lambda V(x), \quad \forall x \in \mathbb{R}^{2} .
\end{gathered}
$$

By periodicity of $f$ in $T$ for any integer $j$

$$
\dot{V}(z, \theta-T j) \leq-\lambda V(z, \theta-T j), \quad \forall(z, \theta) \in \mathbb{R}^{2} .
$$

Denote $V_{j}(x)=V(z, \theta-T j)$, then the invariant sets $\Omega_{j}=$ $\left\{x \in \mathbb{R}^{2}: V_{j}(x) \leq 0\right\}$ are employed in Theorem 1 to establish boundedness of solutions for the system. These sets are illustrated in Fig. 1. More precisely, the set $\Omega_{0}$ and a possible behavior of trajectories in its neighborhood are shown in Fig. 1 ,a, while the intersection of $\Omega_{j}$ for $j=-1,0,1$ is plotted in Fig. 1,b. By construction, the common points in $\cap_{j \in \mathbb{Z}} \Omega_{j}$ form isolated invariant cells (the darkest rectangles in Fig. 1,b), to which all trajectories converge. Note that a passage from one cell to another is impossible since the individual cells are only connected through equilibria. In general, the boundary at which $V_{j}$ changes sign is not a straight line, but can be of an arbitrary shape. The straight lines are chosen here to ease the illustration of the main idea of Theorem 1 and also to motivate the notion of a cell structure.

\section{Problem statement}

The functions proposed in [31] for the analysis of boundedness of trajectories of the system (1) with a scalar periodic state are sign-indefinite with a sign-indefinite derivative. Clearly, such a relaxation of the definiteness of a Lyapunov function might simplify significantly its construction. Usually signindefinite functions with a sign-definite derivative are used to establish instability of an equilibrium of (1), e.g. Chetaev functions [10], [16]. An important observation of [31] is that the combination of "instability" and periodicity leads to boundedness of trajectories: under periodicity the existence of invariant solutions separating the domain of periodic variables, with probably repulsing trajectories around those invariants, implies the existence of a certain cell structure created by periodically repeated invariant solutions, which bounds the 
admissible behavior of the trajectories (see Example 1). A major restriction of the cell structure approach in [31], [24], [48] is that it can only be applied in the case of a scalar periodic component in $x(t)$. As a consequence, the main objective of this work is to extend that approach to a generic multidimensional case.

Inspired by [31], [3], an equivalent characterization of the ISS property for a periodic system on manifolds has been proposed in [18] in terms of ISS-Leonov functions, which are sign-indefinite. In the present work the concept of Leonov functions is further developed for (1) with $x \in \mathbb{R}^{n}$.

For our subsequent derivations, it is convenient to introduce auxiliary sets: $\mathcal{W}=\left(\mathbb{R}^{k} \times \mathcal{S}\right)$ where $\mathcal{S}$ is a $q$-th sphere (a set topologically equivalent to $\mathbb{S}^{q}$ ) such that

$$
\begin{gathered}
\pi \leq|\theta|_{\infty}<2 \pi, \quad \forall x=(z, \theta) \in \mathcal{W}, \\
\mathcal{U}=\bigcup_{r \in \mathbb{Z}_{+}} \mathcal{U}_{r}, \\
\mathcal{U}_{r}=\left\{x=(z, \theta) \in \mathbb{R}^{n}: z=0,|\theta|_{\infty}=2 r \pi, f(x)=0\right\} .
\end{gathered}
$$

Therefore, the set $\mathcal{U}$ includes all equilibria obtained by shifting the one at the origin using the property that, by assumption, $f$ is $2 \pi$-periodic in $\theta$. However, in general, the system (1) may possess other equilibria that do not belong to $\mathcal{U}$.

Definition 1. We say that a $\mathcal{C}^{1}$ function $V: \mathbb{R}^{n} \rightarrow \mathbb{R}$ is a Leonov function for (1) if there exist a constant $g \geq 0$, functions $\alpha \in \mathcal{K}_{\infty}, \psi \in \mathcal{K}$ and a continuous function $\lambda: \mathbb{R} \rightarrow$ $\mathbb{R}$ satisfying $\lambda(0)=0$ and $\lambda(s) s>0$ for all $s \neq 0$, such that

$$
\begin{aligned}
\alpha(|z|)-\psi(|\theta|)-g & \leq V(x), \quad \forall x=(z, \theta) \in \mathbb{R}^{n}, \\
\inf _{x \in \mathcal{W}} V(x) & >0, \sup _{x \in \mathcal{U}} V(x) \leq 0
\end{aligned}
$$

and the following dissipation inequality holds:

$$
\frac{\partial V(x)}{\partial x} f(x) \leq-\lambda(V(x)), \quad \forall x \in \mathbb{R}^{n} .
$$

Roughly speaking, the function $V$ is radially unbounded with respect to the non-periodic variable $z$ and negative definite with respect to the distance to the set $\mathcal{W}$ (it takes positive values on the set $\mathcal{W}$ and negative values at the origin and on the set $\mathcal{U}$ ). Consequently, the conditions (3) can be equivalently formulated as

$$
\begin{gathered}
\alpha(|z|)-\psi^{\prime}\left(|\theta|_{\mathcal{S}}\right)+g^{\prime} \leq V(x), \quad \forall x \in \mathbb{R}^{n}, \\
\sup _{x \in \mathcal{U}} V(x) \leq 0
\end{gathered}
$$

for some $\psi^{\prime} \in \mathcal{K}$ and $g^{\prime} \geq 0$ with $\psi^{\prime}\left(|0|_{\mathcal{S}}\right) \geq g^{\prime}$ (since $V(0) \leq$ $0)$. Note that by construction the set $\mathcal{W}$ cannot contain any equilibrium point of (1) since in such a case the derivative of $V$ would be strictly negative at an equilibrium.

Remark 1. Chetaev's theorem about instability of a set can be formulated as follows [16]: for a $\mathcal{C}^{1}$ function $U: \mathbb{R}^{n} \rightarrow \mathbb{R}$, with $U(x)=0$ for all $x \in \mathcal{Q}$, where $\mathcal{Q} \subset \mathbb{R}^{n}$ is a compact invariant set of (1), if there exists $\epsilon_{0}>0$ such that $\mathcal{U}^{+} \cap$ $\mathcal{B}_{\mathcal{Q}}(\epsilon) \neq \emptyset$ for any $\epsilon \in\left(0, \epsilon_{0}\right]$, where $\mathcal{U}^{+}=\left\{x \in \mathcal{B}_{\mathcal{Q}}\left(\epsilon_{0}\right)\right.$ : $U(x)>0\}$ and $\mathcal{B}_{\mathcal{Q}}(\varepsilon)=\left\{x \in \mathbb{R}^{n}:|x|_{\mathcal{Q}}<\varepsilon\right\}$, and if

$$
\frac{\partial U(x)}{\partial x} f(x)>0, \quad \forall x \in \mathcal{U}^{+}
$$

then (1) is unstable with respect to $\mathcal{Q}$ with the region of repulsion $\mathcal{B}_{\mathcal{Q}}\left(\epsilon_{0}\right)$. Now, take $U(x)=-V(x)$, where $V: \mathbb{R}^{n} \rightarrow \mathbb{R}$ is a Leonov function for (1). Then due to (4) the inequality $\frac{\partial V(x)}{\partial x} f(x)<0$ may be satisfied for $V(x)<0$. In such a case the property (5) can be verified. In addition, if the set $\left\{x \in \mathbb{R}^{n}: V(x)=0\right\}$ (since $V$ is continuous and sign indefinite according to (3) or (6) below, then this set is not empty) contains a compact and invariant set of (1), e.g. an equilibrium, then the existence of a Leonov function $V$ implies instability of (1) at the set $\mathcal{Q}$ with the region of repulsion belonging to $\left\{x \in \mathbb{R}^{n}: V(x) \leq 0\right\}$.

Remark 2. The appearance of the norm $|\cdot|_{\infty}$ in the formulation of a Leonov function is originated by the topology induced by periodicity of $f$ in $\theta$ : replicating the equilibrium at the origin using periodicity in $\theta \in \mathbb{R}^{q}$ creates a set of equilibria located at intersections of the levels $|\theta|_{\infty}=2 j \pi$ for $j \in \mathbb{Z}$.

In order to design a Leonov function for an actual system, the following characterization of existence of a Leonov function may also be employed. This characterization is established by using upper and lower estimates in terms of class $\mathcal{K}$ functions, as often done in the conventional Lyapunov theory. Yet, it is only sufficient for existence of a Leonov function:

Proposition 1. Let $V: \mathbb{R}^{n} \rightarrow \mathbb{R}$ be a $\mathcal{C}^{1}$ function and suppose that there exist functions $\alpha_{1}, \alpha_{2} \in \mathcal{K}_{\infty}, \sigma_{1}, \sigma_{2} \in \mathcal{K}$, and a continuous function $\lambda: \mathbb{R} \rightarrow \mathbb{R}, \lambda(0)=0$ and $\lambda(s) s>0$ for all $s \neq 0$, and scalars $0<g_{1} \leq g_{2} \leq \sigma_{2}(\pi)$, such that for all $x=(z, \theta) \in \mathbb{R}^{n}$

$$
\begin{gathered}
\alpha_{1}(|z|)-\sigma_{1}\left(\left.|| \theta\right|_{\infty}-\pi \mid\right)+g_{1} \leq V(x) \\
\leq \alpha_{2}(|z|)-\sigma_{2}\left(\left.|| \theta\right|_{\infty}-\pi \mid\right)+g_{2},
\end{gathered}
$$

and (4) holds. Then $V$ is a Leonov function for (1).

Proof. By assumption, $g_{1} \leq g_{2} \leq \sigma_{2}(\pi)$. Furthermore, note that at the origin the relations (6) take the form:

$$
0 \geq g_{2}-\sigma_{2}(\pi) \geq V(0) \geq g_{1}-\sigma_{1}(\pi) .
$$

Thus, $g_{1} \leq \sigma_{1}(\pi)$. The restriction $g_{1}>0$ ensures that on the set $\mathcal{W}=\left\{x=(z, \theta) \in \mathbb{R}^{n}:|\theta|_{\infty}=\pi\right\}$ (and in some of its vicinity) the function $V$ takes positive values:

$$
V(x) \geq \alpha_{1}(|z|)+g_{1}>0, \quad \forall x \in \mathcal{W},
$$

while $g_{2} \leq \sigma_{2}(\pi)$ implies that $V(0) \leq 0$, and that

$$
V(x) \leq g_{2}-\sigma_{2}(\pi) \leq 0, \quad \forall x \in \mathcal{U}_{1}
$$

Next, since $\sigma_{2} \in \mathcal{K}$, it is monotonically increasing, and

$$
V(x) \leq g_{2}-\sigma_{2}((2 r-1) \pi) \leq g_{2}-\sigma_{2}(\pi) \leq 0, \quad \forall x \in \mathcal{U}_{r}
$$

for all $r \geq 1$. For any $x \in \mathbb{R}^{n}$ we have from (6):

$$
\begin{aligned}
V(x) & \geq \alpha_{1}(|z|)-\sigma_{1}\left(\left.|| \theta\right|_{\infty}-\pi \mid\right)+g_{1} \\
& \geq \alpha_{1}(|z|)-\tilde{\sigma}_{1}(|\theta|)-g,
\end{aligned}
$$

where existence of some $\tilde{\sigma}_{1} \in \mathcal{K}$ and $g \geq 0$ follows from the relations of the norms $|\cdot|$ and $|\cdot|_{\infty}$, and the demonstrated property $g_{1} \leq \sigma_{1}(\pi)$ (i.e. $V(0) \leq 0$ ). Therefore, all properties of (3) are verified. 


\section{A MULTIVARIABLE CELL STRUCTURE APPROACH}

This section is dedicated to the derivation of a multivariable cell structure approach based on the concept of a Leonov function. Our main result is presented in subsection IV-A. Next, we illustrate how the required conditions can be verified for a special case. Furthermore, we provide two relaxations of our main result, which already have been found very helpful in applications of the proposed cell structure framework, see [41].

\section{A. Main result}

Our main result is as follows. An illustration of the result is given in Example 2 below.

Theorem 2. If for the system (1) under Assumption 1 there exists a Leonov function, then for all $x_{0} \in \mathbb{R}^{n}$ the corresponding trajectories $X\left(t, x_{0}\right)$ are bounded for all $t \geq 0$.

Proof. Denote the set of negative values of $V$ as

$$
\Omega=\left\{x \in \mathbb{R}^{n}: V(x) \leq 0\right\} .
$$

From the definition of the set $\Omega$ and the lower bound of the function $V$ in (3) we obtain:

$$
\alpha(|z|) \leq g+\psi(|\theta|), \quad \forall x=(z, \theta) \in \Omega .
$$

Recall the sets $\mathcal{U}$ and $\mathcal{W}$ defined in (2), and that $|\theta| \leq q|\theta|_{\infty}$. Then from the definition of a Leonov function, see (3), the set $\mathcal{U}$ belongs to $\Omega$, while $\mathcal{W} \notin \Omega$. Furthermore, the set $\Omega$ can be decomposed into two parts:

$$
\Omega=\Omega^{\prime} \cup \Omega^{\prime \prime}
$$

The first of these sets,

$$
\Omega^{\prime} \subset\left\{x \in \mathbb{R}^{n}:|z| \leq \alpha^{-1}(\tilde{g}),|\theta|_{\infty} \leq c\right\}
$$

is a compact set containing the origin for some $c \in(\pi, 2 \pi)$, i.e. $\mathcal{U}_{0} \subset \Omega^{\prime}$, with $g \leq \tilde{g} \leq g+\psi(q c)$ (the constant $\tilde{g}$ evaluates the upper bound on $|z|$ after which the growing terms in $z$ will force $V$ to take positive values for any $\left.|\theta|_{\infty} \leq c\right)$. The second set is defined as

$$
\Omega^{\prime \prime} \subset\left\{x \in \mathbb{R}^{n}:|\theta|_{\infty}>c\right\} .
$$

Both these sets are non-empty since $0 \in \Omega^{\prime}$ and $\mathcal{U}_{r} \in \Omega^{\prime \prime}$ for $r \geq 1$ due to (3). Furthermore, the set $\Omega^{\prime \prime}$ is unbounded. In addition, the subsets $\Omega^{\prime}$ and $\Omega^{\prime \prime}$ are separated by the set $\mathcal{W}$ (the definition of which determines the value of $c$ ).

Denote $V(t)=V\left(X\left(t, x_{0}\right)\right)$ for any $x_{0} \in \mathbb{R}^{n}$, then under the conditions of the theorem we have:

$$
\dot{V}(t)+\lambda(V(t)) \leq 0, \quad \forall t \geq 0 .
$$

Thus, clearly, $V(t)$ is strictly decreasing while $X\left(t, x_{0}\right) \in \mathbb{R}^{n}$ $\Omega$, i.e. while $V(t)>0$. Therefore, if $x_{0} \in \Omega$ then $X\left(t, x_{0}\right) \in$ $\Omega$ for all $t \geq 0$ and the set $\Omega$ is forward invariant for (1). Conversely, if $x_{0} \in \mathbb{R}^{n} \backslash \Omega$ then there exists $0 \leq T_{x_{0}}<+\infty$ such that $X\left(T_{x_{0}}, x_{0}\right) \in \Omega$ and, by invariance, the trajectory remains in this set for all $t \geq T_{x_{0}}$. Thus, the set $\Omega$ is globally attractive and forward invariant. Note that this property does not imply any kind of stability since $\Omega$ may be unbounded (it could also be interpreted as instability of a set containing $\mathcal{W}$ with the domain of repulsion $\mathbb{R}^{n} \backslash \Omega$ ).

To establish stability, we exploit the periodicity of (1). Denote by $j=\left[j_{1}, \ldots, j_{q}\right]$ a multi-index vector, where $j_{i} \in \mathbb{Z}$ for all $i=1, \ldots, q$. Introduce the new variable $x_{j}=x-\left[\begin{array}{c}0_{k} \\ 2 \pi j\end{array}\right]$ and the function $V_{j}(x)=V\left(x-\left[\begin{array}{c}0_{k} \\ 2 \pi j\end{array}\right]\right)$ for any such multi-index vector $j$ (i.e. $V_{0}(x)=V(x)$ ), where $0_{k}$ is the zero vector of dimension $k$. Hence

$$
V_{j}(x)=V_{j}\left(x_{j}+\left[\begin{array}{c}
0_{k} \\
2 \pi j
\end{array}\right]\right)=V\left(x_{j}\right) .
$$

Furthermore, by $2 \pi$-periodicity of $f$ in $\theta$,

$$
\dot{x}_{j}(t)=f(x(t))=f\left(x_{j}(t)\right), \quad \forall t \geq 0 .
$$

Therefore, taking into account the properties substantiated for (1) and $V$, we obtain that the set $\left\{x_{j} \in \mathbb{R}^{n}: V\left(x_{j}\right) \leq 0\right\}$ is globally attractive and forward invariant, which in the original coordinates $x$ implies these properties for the set

$$
\begin{aligned}
\Omega_{j} & =\left\{x \in \mathbb{R}^{n}: V_{j}(x) \leq 0\right\} \\
& \left.=\left\{x \in \mathbb{R}^{n}: V\left(x-\left[\begin{array}{c}
0_{k} \\
2 \pi j
\end{array}\right]\right) \leq 0\right)\right\} .
\end{aligned}
$$

Using similar arguments as for $\Omega$ it is possible to show that $\Omega_{j}=\Omega_{j}^{\prime} \cup \Omega_{j}^{\prime \prime}$, where

$$
\begin{gathered}
\Omega_{j}^{\prime} \subset\left\{x \in \mathbb{R}^{n}:|z| \leq \alpha^{-1}(\tilde{g}),|\theta-2 \pi j|_{\infty} \leq c\right\}, \\
\Omega_{j}^{\prime \prime} \subset\left\{x \in \mathbb{R}^{n}:|\theta-2 \pi j|_{\infty}>c\right\} .
\end{gathered}
$$

Since the sets $\Omega_{j}$ are forward invariant, any trajectory $X\left(t, x_{0}\right)$ has to belong asymptotically to the intersection of these sets:

$$
\bigcap_{j} \Omega_{j}=\bigcap_{j}\left(\Omega_{j}^{\prime} \cup \Omega_{j}^{\prime \prime}\right)=\bigcap_{j} \Omega_{j}^{\prime} \cup \bigcap_{j} \Omega_{j}^{\prime \prime} \cup \Xi,
$$

where $\Xi$ belongs to the union of all possible intersections of the form $\Omega_{j_{1}}^{\prime} \cap \Omega_{j_{2}}^{\prime \prime}$ with $j_{1} \neq j_{2}$ :

$$
\Xi \subset \bigcup_{j}\left(\Omega_{j}^{\prime} \cap \bigcup_{k \neq j} \Omega_{k}^{\prime \prime}\right)
$$

Next, we show that $\bigcap_{j} \Omega_{j}$ is composed of compact isolated subsets. At first, we note that since $c \in(\pi, 2 \pi)$, then $\bigcap_{j} \Omega_{j}^{\prime}=$ $\bigcup_{j} \Theta_{j}$, where $\Theta_{j} \subset \Omega_{j}^{\prime}$ is formed by compact isolated sets due to the fact that all $\Omega_{j}^{\prime}$ possess the same property. Furthermore, by definition $\bigcap_{j} \Omega_{j}^{\prime \prime}=\emptyset$ due to the fact that $\Omega_{j}^{\prime \prime} \subset \mathbb{R}^{n} \backslash \Upsilon_{j}$ with $\Upsilon_{j}=\left\{x \in \mathbb{R}^{n}:|\theta-2 \pi j|_{\infty} \leq c\right\}$, then $\bigcap_{j} \Omega_{j}^{\prime \prime} \subset \bigcap_{j} \mathbb{R}^{n} \backslash$ $\Upsilon_{j}=\mathbb{R}^{n} \backslash \bigcup_{j} \Upsilon_{j}=\mathbb{R}^{n} \backslash \mathbb{R}^{n}=\emptyset$. Since $\left(0_{k}, 2 \pi j_{1}\right) \in \mathcal{U} \cap$ $\Omega_{j_{1}}^{\prime}$ for any $j_{1}$ by definition of both sets, and $\mathcal{U} \backslash \Omega_{j_{2}}^{\prime} \subset \Omega_{j_{2}}^{\prime \prime}$ due to $c \in(\pi, 2 \pi)$. These two facts imply that $\left(0_{k}, 2 \pi j_{1}\right) \in \Omega_{j_{2}}^{\prime \prime}$ for any $j_{1} \neq j_{2}$. Consequently, $\Omega_{j_{1}}^{\prime} \cap \Omega_{j_{2}}^{\prime \prime} \neq \emptyset$ for any multiindex vectors $j_{1} \neq j_{2}$, which implies that $\Xi \subset \bigcup_{j} \Omega_{j}^{\prime} \cap \mathcal{R}_{j}$ since $c \in(\pi, 2 \pi)$, where $\mathcal{R}_{j}=\left\{x \in \mathbb{R}^{n}:|\theta-2 \pi j|_{\infty}<\pi\right\}$. Therefore, we have shown that

$$
\bigcap_{j} \Omega_{j} \subset\left(\bigcup_{j} \Omega_{j}^{\prime} \cap \mathcal{R}_{j}\right) \cup\left(\bigcup_{j} \Theta_{j}\right),
$$




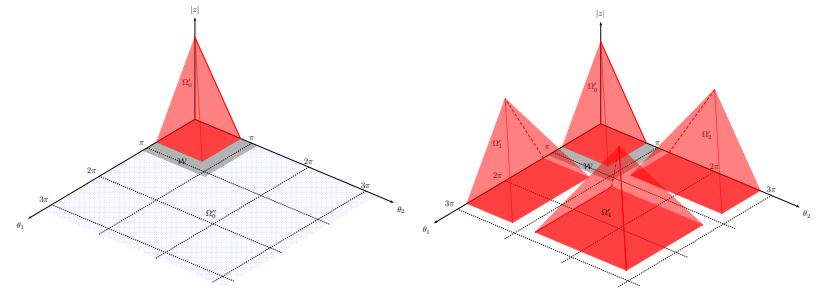

Figure 2. Structure of the set $\Omega$

where for each $j$ the sets $\Omega_{j}^{\prime} \cap \mathcal{R}_{j}$ and $\Theta_{j}$ are compact and isolated; for any neighboring $\Omega_{j_{1}}^{\prime} \cap \mathcal{R}_{j_{1}} \subset \Omega_{j_{2}}^{\prime \prime}$ and $\Theta_{j_{2}} \subset \Omega_{j_{2}}^{\prime}$ there is no intersection by construction $\left(\Omega_{j_{2}}^{\prime} \cap \Omega_{j_{2}}^{\prime \prime}=\emptyset\right)$. Hence, the compact sets in $\bigcap_{j} \Omega_{j}$ form a kind of cell cover of $\{x \in$ $\left.\mathbb{R}^{n}:|z| \leq \alpha^{-1}(\tilde{g})\right\}$. Recall that for all multi-index vectors $j$ the corresponding sets $\Omega_{j}$ are globally attracting and forward invariant. Take any $x_{0} \in \mathbb{R}^{n}$, then $X\left(t, x_{0}\right)$ asymptotically enters and stays in a cell $\Omega_{j}^{\prime}$ for some $j$. Hence, for any $x_{0} \in$ $\mathbb{R}^{n}$ the corresponding solution $X\left(t, x_{0}\right)$ is bounded for all $t \geq 0$.

Example 2. For an illustration of the conditions of Theorem 2 , let us consider an example of (1) with $k=1$ and $q=2$, then $x=(z, \theta) \in \mathbb{R}^{3}$ and $f: \mathbb{R}^{3} \rightarrow \mathbb{R}^{3}$ satisfies Assumption 1 . Assume that there is a Leonov function $V: \mathbb{R}^{n} \rightarrow \mathbb{R}$ for some $g \geq 0$, functions $\alpha \in \mathcal{K}_{\infty}, \psi \in \mathcal{K}$ and a continuous function $\lambda: \mathbb{R} \rightarrow \mathbb{R}$ satisfying (3) and (4) for all $x=(z, \theta) \in \mathbb{R} \times \mathbb{R}^{2}$. Furthermore, let $\mathcal{W}=\left\{x=(z, \theta) \in \mathbb{R}^{3}:|\theta|_{\infty}=\pi\right\}$. Then a possible form of the set $\Omega=\Omega_{0}=\Omega_{0}^{\prime} \cup \Omega_{0}^{\prime \prime}$ is shown in the left part of Fig. 2, where the compact set $\Omega_{0}^{\prime}$ is represented by a red pyramid, while the unbounded set $\Omega_{0}^{\prime \prime}$ is just illustrated by its blue projection on the surface $z=0$. The gray-colored area corresponds to the projection of the set $\left\{x \in \mathbb{R}^{n}: V(x)>0\right\}$ to the surface $z=0$. This set also contains $\mathcal{W}$ and separates $\Omega_{0}^{\prime}$ from $\Omega_{0}^{\prime \prime}$. By applying periodicity and constructing the sets $\Omega_{j}=\Omega_{j}^{\prime} \cup \Omega_{j}^{\prime \prime}$, after their intersection we obtain the set of red pyramids shown in the right part of Fig. 2, which now form a kind of multivariable cell cover of the globally attracting and forward invariant set of (1).

Remark 3. Comparing the results given in theorems 1 and 2 we conclude that there is one property imposed in the latter case to the Leonov function $V(x)$, which is not satisfied in the scalar one for the function $V_{0}(x)=x^{\top} H x$ :

$$
\inf _{x \in \mathcal{W}} V(x)>0 .
$$

This property is crucial for construction of a cell cover for $q>1$ with isolated (globally attracting and invariant) cells. It also explains why a straightforward generalization of the result of Theorem 1 to the multi-dimensional case is complicated, see also the discussion in [35].

\section{B. Equivalent conditions and relaxations}

Theorem 2 provides a very general result on application of the theory of Leonov functions. To illustrate and clarify the idea of the approach, equivalent conditions for a special case are developed below.
Corollary 1. Suppose that there exist $\mathcal{C}^{1}$ functions $W: \mathbb{R}^{n} \rightarrow$ $\mathbb{R}$ and $\varphi: \mathbb{R}^{n} \rightarrow \mathbb{R}$, functions $\alpha_{1}, \alpha_{2} \in \mathcal{K}_{\infty}$, constants $\lambda>0$ and $\bar{a}_{i} \geq \underline{a}_{i}>0, \bar{c}_{i} \geq \underline{c}_{i} \geq 0, \underline{b}_{i} \geq \bar{b}_{i}>0, i=1, \ldots, q$, such that for all $x \in \mathbb{R}^{n}$

$$
\begin{aligned}
\alpha_{1}(|z|) & \leq W(x) \leq \alpha_{2}(|z|), \\
\sum_{i=1}^{q}\left(\underline{a}_{i} \theta_{i}^{2}-\underline{b}_{i}\left|\theta_{i}\right|+\underline{c}_{i}\right) & \leq \varphi(x) \leq \sum_{i=1}^{q}\left(\bar{a}_{i} \theta_{i}^{2}-\bar{b}_{i}\left|\theta_{i}\right|+\bar{c}_{i}\right), \\
\frac{\partial V(x)}{\partial x} & f(x)+\lambda V(x) \leq 0,
\end{aligned}
$$

where $V(x)=W(x)-\varphi(x)$ and $x=(z, \theta)$. Then $X\left(t, x_{0}\right)$ of (1) is bounded for any $x_{0} \in \mathbb{R}^{n}$ and $t \geq 0$ provided that

$$
\begin{gathered}
\underline{a}_{\min } \pi+\sum_{i=1}^{q} \underline{c}_{i} \pi^{-1}<q \underline{b}_{\max }<2 \underline{a}_{\min } \pi+0.5 \sum_{i=1}^{q} \underline{c}_{i} \pi^{-1} \\
q^{2} \bar{a}_{\max } \pi+\sum_{i=1}^{q} \bar{c}_{i} \pi^{-1}<\bar{b}_{\min }<2 q^{2} \bar{a}_{\max } \pi+0.5 \sum_{i=1}^{q} \bar{c}_{i} \pi^{-1}
\end{gathered}
$$

where $\underline{a}_{\min }=\min _{1 \leq i \leq q} \underline{a}_{i}, \underline{b}_{\max }=\max _{1 \leq i \leq q} \underline{b}_{i}, \bar{a}_{\max }=$ $\max _{1 \leq i \leq q} \bar{a}_{i}$ and $\bar{b}_{\min }=\min _{1 \leq i \leq q} \bar{b}_{i}$.

Proof. The claim is established by applying Theorem 2 and Proposition 1. Note that (4) is satisfied by assumption. Moreover, under the standing hypothesis,

$$
\alpha_{1}(|z|)-\varphi(x) \leq V(x) \leq \alpha_{2}(|z|)-\varphi(x) .
$$

Thus, by invoking Proposition 1, it suffices to show that there exist some $\sigma_{1}, \sigma_{2} \in \mathcal{K}$ and some $0<g_{1} \leq g_{2} \leq \sigma_{2}(\pi)$, such that

$$
\sigma_{2}\left(\left.|| \theta\right|_{\infty}-\pi \mid\right)-g_{2} \leq \varphi(x) \leq \sigma_{1}\left(\left.|| \theta\right|_{\infty}-\pi \mid\right)-g_{1} .
$$

Note that

$$
\begin{gathered}
\sum_{i=1}^{q}\left(\bar{a}_{i} \theta_{i}^{2}-\bar{b}_{i}\left|\theta_{i}\right|+\bar{c}_{i}\right) \leq \sum_{i=1}^{q}\left(\bar{a}_{\max } \theta_{i}^{2}-\bar{b}_{\min }\left|\theta_{i}\right|+\bar{c}_{i}\right) \\
=\bar{a}_{\max }|\theta|^{2}+\sum_{i=1}^{q}\left(-\bar{b}_{\min }\left|\theta_{i}\right|+\bar{c}_{i}\right) \\
\leq q^{2} \bar{a}_{\max }|\theta|_{\infty}^{2}-\bar{b}_{\min }|\theta|_{\infty}+\sum_{i=1}^{q} \bar{c}_{i}:=\bar{f}\left(|\theta|_{\infty}\right)
\end{gathered}
$$

and

$$
\begin{gathered}
\sum_{i=1}^{q}\left(\underline{a}_{i} \theta_{i}^{2}-\underline{b}_{i}\left|\theta_{i}\right|+\underline{c}_{i}\right) \geq \sum_{i=1}^{q}\left(\underline{a}_{\min } \theta_{i}^{2}-\underline{b}_{\max }\left|\theta_{i}\right|+\underline{c}_{i}\right) \\
=\underline{a}_{\min }|\theta|^{2}+\sum_{i=1}^{q}\left(-\underline{b}_{\max }\left|\theta_{i}\right|+\underline{c}_{i}\right) \\
\geq \underline{a}_{\min }|\theta|_{\infty}^{2}-q \underline{b}_{\max }|\theta|_{\infty}+\sum_{i=1}^{q} \underline{c}_{i}:=\underline{f}\left(|\theta|_{\infty}\right) .
\end{gathered}
$$

Consequently,

$$
\underline{f}\left(|\theta|_{\infty}\right) \leq \varphi(x) \leq \bar{f}\left(|\theta|_{\infty}\right) .
$$


Moreover, under the introduced restrictions the following properties are satisfied for $\underline{f}$ :

$$
\begin{gathered}
\sum_{i=1}^{q} \underline{c}_{i}=\underline{f}(0) \geq 0, \\
\underline{a}_{\min } \pi+\sum_{i=1}^{q} \underline{c}_{i} \pi^{-1}<q \underline{b}_{\max } \Rightarrow \underline{f}(\pi)<0, \\
2 \underline{a}_{\min } \pi+0.5 \sum_{i=1}^{q} \underline{c}_{i} \pi^{-1}>q \underline{b}_{\max } \Rightarrow \underline{f}(2 \pi)>0 .
\end{gathered}
$$

Furthermore, $2 \pi^{2} \underline{a}_{\min }>\sum_{i=1}^{q} \underline{c}_{i}$ is a sufficient condition for existence of $\underline{b}_{\max }$, which is satisfied due to the imposed assumptions. Similarly for $\bar{f}$ :

$$
\begin{gathered}
\sum_{i=1}^{q} \bar{c}_{i}=\bar{f}(0) \geq 0, \\
q^{2} \bar{a}_{\max } \pi+\sum_{i=1}^{q} \bar{c}_{i} \pi^{-1}<\bar{b}_{\min } \Rightarrow \bar{f}(\pi)<0, \\
2 q^{2} \bar{a}_{\max } \pi+0.5 \sum_{i=1}^{q} \bar{c}_{i} \pi^{-1}>\bar{b}_{\text {min }} \Rightarrow \bar{f}(2 \pi)>0
\end{gathered}
$$

and the inequality $2 \pi^{2} q^{2} \bar{a}_{\max }>\sum_{i=1}^{q} \bar{c}_{i}$ is satisfied by assumptions. From these properties of $\frac{f}{}$ and $\bar{f}$ we can conclude that the required functions $\sigma_{1}, \bar{\sigma}_{2} \in \mathcal{K}$ and constants $0<g_{1} \leq g_{2} \leq \sigma_{2}(\pi)$ exist, completing the proof (for example, these functions can be selected in the form $\sigma_{1}(s)=\ell_{1} \max \left\{\ell_{2} s, s^{2}\right\}$ and $\sigma_{2}(s)=\ell_{3} \min \left\{\ell_{4} s, s^{2}\right\}$ for some $\ell_{i}>0$ for $\left.i=1,2,3,4\right)$.

Another possibility to relax the conditions of Theorem 2 is based on the observation that, in the proof of the main result, the property (4) is used only for $V(x) \geq 0$ :

Corollary 2. If for the system (1) under Assumption 1 there exists a Leonov function $V: \mathbb{R}^{n} \rightarrow \mathbb{R}$ such that the inequality (4) is verified only for $x \in\left(\mathbb{R}^{n} \backslash \Omega_{0}\right) \cap\left(\mathcal{Z} \cup \Omega_{\varepsilon}\right)$, where $\Omega_{\varepsilon}:=\left\{x \in \mathbb{R}^{n}: V(x) \leq \varepsilon\right\}$ and $\mathcal{Z}=\left\{x=(z, \theta) \in \mathbb{R}^{n}:\right.$ $|z| \geq \zeta\}$ for some $\varepsilon>0$ and $\zeta \in \mathbb{R}_{+}$, then for all $x_{0} \in \mathbb{R}^{n}$ the corresponding trajectories $X\left(t, x_{0}\right)$ are bounded for all $t \geq 0$.

Proof. Note that the inequality (4) does not allow to make any conclusion about the behavior of the Leonov function $V(x)$ while $x \in \Omega=\Omega_{0}$. Hence, limiting the validity of (4) to the set $\mathbb{R}^{n} \backslash \Omega$ is not restrictive at all. Next, it is necessary to take into consideration the set $\mathcal{C} \subset \mathbb{R}^{n} \backslash\left(\Omega_{\varepsilon} \cup \mathcal{Z}\right)$. Clearly on this set (4) is not obligatorily satisfied. But since $\Omega \cap \mathcal{C}=\emptyset$ for any $\varepsilon>0$ it follows that the set $\Omega=\Omega^{\prime} \cup \Omega^{\prime \prime}$ preserves its property of forward invariance for (1) and local attractiveness established in Theorem 2. Note that in the present case only local attractiveness can be claimed for $\Omega$ since the inequality (4) is not necessarily satisfied on $\mathcal{C}$ for $V(x)>0$. Therefore, the system behavior is not specified there and a trajectory may stay in $\mathcal{C}$ (or travel between isolated subsets of $\mathcal{C}$ ). Since $\mathcal{C} \subset \mathbb{R}^{n} \backslash \Omega$, then $\mathcal{C} \subset\left\{x=(z, \theta) \in \mathbb{R}^{n}: \underline{c} \leq|\theta|_{\infty} \leq \bar{c}\right\}$ for some $\underline{c} \in(0, \pi)$ and $\bar{c} \in(\pi, 2 \pi)$ by definition of the set $\Omega$ (see the proof of Theorem 2, with $\underline{c}<c \leq \bar{c}$ where $c \in(\pi, 2 \pi)$ is used in the definition of the sets $\Omega^{\prime}$ and $\left.\Omega^{\prime \prime}\right)$. Consequently, taking into account the set $\mathcal{Z}$ we obtain that

$$
\mathcal{C} \subset\left\{x=(z, \theta) \in \mathbb{R}^{n}:|z|<\zeta, \underline{c} \leq|\theta|_{\infty} \leq \bar{c}\right\},
$$

which is a bounded set. Next we show that any trajectory, which enters in $\mathcal{C}$ or even travels between isolated subsets of $\mathcal{C}$, cannot leave a bounded domain containing $\mathcal{C}$. Indeed, if a trajectory reaches either the set with $|\theta|_{\infty}>\bar{c}$ or $|\theta|_{\infty}<\underline{c}$, then it is in the set $\Omega$, which is forward invariant and the behavior of $X\left(t, x_{0}\right)$ in this set is analyzed later. Therefore, assume that $|\theta|_{\infty}$ stays bounded by $\underline{c}$ and $\bar{c}$. Then from the definition of a Leonov function and its lower bound $(\alpha(|z|)-$ $\psi(|\theta|)-g \leq V(x)$ for all $x=(z, \theta) \in \mathbb{R}^{n}$ and some $g \geq 0$, $\alpha \in \mathcal{K}_{\infty}, \psi \in \mathcal{K}$ ) and also due to the imposed assumptions on the domain of validity of (4), there is a finite constant $\Xi \geq \max \left\{\zeta, \alpha^{-1}(\psi(q \bar{c})+g)\right\}$ such that

$$
V(x)>0, \frac{\partial V(x)}{\partial x} f(x) \leq-\lambda(V(x))
$$

provided that $x \in\left\{x=(z, \theta) \in \mathbb{R}^{n}:|z| \geq \Xi, \underline{c} \leq|\theta|_{\infty} \leq\right.$ $\bar{c}\}$, which implies upper boundedness of $V(x)$ in this set and consequently boundedness of $z$. Therefore, the union $\Omega^{\prime} \cup$ $\Omega^{\prime \prime} \cup \mathcal{C}$ only contains forward invariant and globally attracting sets for (1). In addition, all these sets are isolated, and if a trajectory $X\left(t, x_{0}\right)$ enters and remains in $\mathcal{C}$, then this implies boundedness of the trajectory (since the set $\mathcal{C}$ is bounded). Hence, the set $\Omega=\Omega^{\prime} \cup \Omega^{\prime \prime}$ preserves its meaning established in Theorem 2. Consequently, repeating the arguments of the proof of Theorem 2 (with introduction of the sets $\Omega_{j}=\Omega_{j}^{\prime} \cup$ $\left.\Omega_{j}^{\prime \prime}\right)$ leads to the conclusion that the trajectories $X\left(t, x_{0}\right)$ are bounded for all $x_{0} \in \mathbb{R}^{n}$ and all $t \geq 0$.

By definition $\Omega \subset \Omega_{\varepsilon}$ and since $\Omega=\Omega^{\prime} \cup \Omega^{\prime \prime}$, then $\Omega_{\varepsilon}=$ $\Omega_{\varepsilon}^{\prime} \cup \Omega_{\varepsilon}^{\prime \prime}$, where $\Omega^{\prime} \subset \Omega_{\varepsilon}^{\prime}$ and $\Omega^{\prime \prime} \subset \Omega_{\varepsilon}^{\prime \prime}$. Therefore, the sets $\Omega_{\varepsilon}^{\prime}$ and $\Omega_{\varepsilon}^{\prime \prime}$ are separated for a sufficiently small $\varepsilon>0$. We can further relax the conditions of Corollary 2 by requiring (4) to be verified only on one of the sets $\Omega_{\varepsilon}^{\prime}$ or $\Omega_{\varepsilon}^{\prime \prime}$ instead of $\Omega_{\varepsilon}$, which may be important in an application.

Corollary 3. Suppose that for the system (1) under Assumption 1 there exists a Leonov function $V: \mathbb{R}^{n} \rightarrow \mathbb{R}$ such that the inequality (4) is verified for one of these options:

a) $x \in\left(\mathbb{R}^{n} \backslash \Omega_{0}\right) \cap\left(\mathcal{Z} \cup \Omega_{\varepsilon}^{\prime}\right)$ and $\sup _{\theta \in \mathbb{R}^{q}} \psi(|\theta|)<+\infty$,

b) $x \in\left(\mathbb{R}^{n} \backslash \Omega_{0}\right) \cap\left(\mathcal{Z} \cup \Omega_{\varepsilon}^{\prime \prime}\right)$, where $\Omega_{\varepsilon}=\Omega_{\varepsilon}^{\prime} \cup \Omega_{\varepsilon}^{\prime \prime}=\left\{x \in \mathbb{R}^{n}: V(x) \leq \varepsilon\right\}$ and $\mathcal{Z}=\{x=$ $\left.(z, \theta) \in \mathbb{R}^{n}:|z| \geq \zeta\right\}$ for some $\varepsilon>0$ and $\zeta \in \mathbb{R}_{+}$. Then for all $x_{0} \in \mathbb{R}^{n}$ the corresponding trajectories $X\left(t, x_{0}\right)$ are bounded for all $t \geq 0$.

Proof. By repeating the arguments of Corollary 2 we can substantiate local attractiveness and forward invariance of the sets $\Omega^{\prime}$ or $\Omega^{\prime \prime}$ for the cases $a$ ) and $b$ ), respectively. In these cases we have no conclusion about the behavior of the solutions $X\left(t, x_{0}\right)$ in the sets $\mathcal{C}^{\prime} \subset \mathbb{R}^{n} \backslash\left(\Omega_{\varepsilon}^{\prime} \cup \mathcal{Z}\right)$ or $\mathcal{C}^{\prime \prime} \subset \mathbb{R}^{n} \backslash\left(\Omega_{\varepsilon}^{\prime \prime} \cup \mathcal{Z}\right)$, correspondingly, which by construction admit the following restrictions:

$$
\begin{aligned}
& \mathcal{C}^{\prime} \subset\left\{x=(z, \theta) \in \mathbb{R}^{n}:|z|<\zeta,|\theta|_{\infty} \geq c\right\} \\
& \mathcal{C}^{\prime \prime} \subset\left\{x=(z, \theta) \in \mathbb{R}^{n}:|z|<\zeta,|\theta|_{\infty} \leq c\right\}
\end{aligned}
$$


for some $c \in(\pi, 2 \pi)$. The set $\mathcal{C}^{\prime \prime}$ is obviously bounded and $\sup _{|\theta|_{\infty} \leq c} \psi(\theta) \leq \psi(q c)$. The set $\mathcal{C}^{\prime}$ is unbounded in $\theta$, but due to the additional restriction imposed on $\psi$ there is $\rho>0$ such that $\sup _{|\theta|_{\infty} \geq c} \psi(\theta) \leq \rho$. Hence, from the definition of a Leonov function and the imposed assumptions there are finite constants $\Xi^{\prime} \geq \max \left\{\zeta, \alpha^{-1}(\rho+g)\right\}$ and $\Xi^{\prime \prime} \geq \max \left\{\zeta, \alpha^{-1}(\psi(q c)+g)\right\}$ for the cases $\left.a\right)$ and $\left.b\right)$, respectively, such that

$$
V(x)>0, \frac{\partial V(x)}{\partial x} f(x) \leq-\lambda(V(x))
$$

provided that $x \in\left\{x=(z, \theta) \in \mathbb{R}^{n}:|z| \geq \Xi^{\prime},|\theta|_{\infty} \geq c\right\}$, respectively that $x \in\left\{x=(z, \theta) \in \mathbb{R}^{n}:|z| \geq \Xi^{\prime \prime},|\theta|_{\infty} \leq\right.$ $c\}$. This implies upper boundedness of $V(x)$ in both sets $\mathcal{C}^{\prime}$ and $\mathcal{C}^{\prime \prime}$, and consequently boundedness of $z$ :

$$
\begin{aligned}
\alpha(|z|) & \leq V(x)+\psi(|\theta|)+g \\
& \leq V(x)+\max \{\rho, \psi(q c)\}+g .
\end{aligned}
$$

Therefore, in both cases $a$ ) and $b$ ), the unions $\Omega^{\prime} \cup \mathcal{C}^{\prime}$ and $\Omega^{\prime \prime} \cup \mathcal{C}^{\prime \prime}$ contain forward invariant and globally attracting sets for (1). Next, the desired conclusion follows by repeating the steps of Theorem 2 (replacing in the arguments $\Omega^{\prime \prime}$ with $\mathcal{C}^{\prime}$ or $\Omega^{\prime}$ with $\left.\mathcal{C}^{\prime \prime}\right)$.

\section{A RELATION WITH INPUT-TO-STATE STABILITY}

In addition to stability, robustness properties with respect to exogenous disturbances are also of great relevance in many control applications. For the class of periodic systems considered in the present paper, robustness in terms of the ISS property has been established in [18] via the notion of an ISS-Leonov function. However, in [18] it is assumed that the states evolve on a manifold $M$, while in the present paper it is assumed that they evolve in $\mathbb{R}^{n}$.

We next show that a Leonov function as proposed in the present paper is closely related to an ISS-Leonov function as introduced in [18] and, thus, with minor additional requirements, existence of a Leonov function also allows to establish the ISS property for (1). To this end, it is more convenient to characterize a Leonov function via the conditions (4) and (6) rather than the more generic conditions in Definition 1. Furthermore, we consider the following system with an external input:

$$
\dot{x}(t)=F(x(t), d(t)),
$$

where $x(t) \in \mathbb{R}^{n}$ and $d(t) \in \mathbb{R}^{m}$ (the input $d(\cdot)$ is a locally essentially bounded and measurable signal) for $t \geq 0, f(x)=$ $F(x, 0)$ and Assumption 1 is satisfied. In such a case any solution $X\left(t, x_{0}, d\right)$ of (7) for an initial condition $x_{0} \in \mathbb{R}^{n}$ and an input $d(\cdot)$ can be embedded in a manifold $M=\mathbb{R}^{k} \times \mathbb{S}^{q}$ by a $2 \pi$-projection of the part of the state $\theta$.

Recall from [18] that for a set $\mathcal{A} \subset \mathbb{R}^{n}$, a $\mathcal{C}^{1}$ function $\mathcal{V}: \mathbb{R}^{n} \rightarrow \mathbb{R}$ is a practical ISS-Leonov function for (7) if there exist functions $\vartheta_{1}, \vartheta_{2}, \sigma, \gamma \in \mathcal{K}_{\infty}$, a continuous function $\lambda: \mathbb{R} \rightarrow \mathbb{R}, \lambda \in \mathcal{K}_{\infty}$ for nonnegative arguments, and scalars $r \geq 0, \nu \geq 0$ such that for all $x=(z, \theta) \in \mathbb{R}^{n}$ and $d \in \mathbb{R}^{m}$

$$
\vartheta_{1}\left(|x|_{\mathcal{A}}\right)-\sigma(|\theta|) \leq \mathcal{V}(x) \leq \vartheta_{2}\left(|x|_{\mathcal{A}}+\nu\right),
$$

and the following dissipation inequality holds:

$$
\frac{\partial \mathcal{V}(x)}{\partial x} F(x, d) \leq-\lambda(\mathcal{V}(x))+\gamma(|d|)+r .
$$

If the latter inequality holds for $r=0$, then $\mathcal{V}$ is said to be an ISS-Leonov function.

Assumption 2. Let a set $\mathcal{A} \subset \mathbb{R}^{n}$ contain all $\alpha$ - and $\omega$ limit sets of (1) and its projection on $M$ be compact and decomposable (in the sense of [3]).

It has been shown in [18] that under the hypothesis given in Assumption 2, the existence of a practical ISS-Leonov function is necessary and sufficient for ISS of (7) with respect to the set $\mathcal{A}$. Then the following result can be formulated for a Leonov function.

Proposition 2. Consider a Leonov function $V: \mathbb{R}^{n} \rightarrow \mathbb{R}$ satisfying the restrictions of Proposition 1. Let for $\mathcal{V}(x)=$ $V(x)+\tau$ with some $\tau \geq 0$, and assume that (4) can be strengthened to (9) with a radially unbounded function $\lambda$ for positive arguments, and that the inequalities

$$
\begin{aligned}
& \vartheta_{1}\left(|x|_{\mathcal{A}}\right)-\sigma(|\theta|) \leq \alpha_{1}(|z|)-\sigma_{1}\left(\left.|| \theta\right|_{\infty}-\pi \mid\right)+g_{1}+\tau \\
& \alpha_{2}(|z|)-\sigma_{2}\left(\left.|| \theta\right|_{\infty}-\pi \mid\right)+g_{2}+\tau \leq \vartheta_{2}\left(|x|_{\mathcal{A}}+\nu\right)
\end{aligned}
$$

are verified for all $x=(z, \theta) \in \mathbb{R}^{n}$. Then $\mathcal{V}$ is a practical ISS-Leonov function for (7) and a set $\mathcal{A} \subset \mathbb{R}^{n}$.

Proof. The result can be deduced by a straightforward application of Definition 1 and the restrictions given in propositions 1 and the current one.

Note that if $\left\{x=(z, \theta) \in \mathbb{R}^{n}: z=0\right\} \subset \mathcal{A}$ then there is $\tau \geq 0$ such that (10) is verified, and by taking $\nu>0$ large enough the fulfillment of the inequality (11) can also be guaranteed.

Corollary 4. Let assumptions 1 and 2 be satisfied together with the hypothesis of Proposition 2. Then the system (7) is ISS with respect to the set $\mathcal{A}$.

Proof. The result is a direct consequence of Proposition 2 and the main result of [18].

It is important to stress here that the ISS property is obtained on the manifold $M$ only (when boundedness of the variable $\theta$ comes directly from the structure of $M$ ). The fact that $V$ is also a Leonov function as introduced in Definition 1 implies, via the claim of Theorem 2 , that for $d(t) \equiv 0$ the solutions of (1) are bounded in $\mathbb{R}^{n}$.

\section{AN ACADEMIC EXAMPLE}

We demonstrate the usefulness of the proposed approach by performing a global analysis of boundedness of solutions of the following system:

$$
\begin{aligned}
& \dot{z}=F z+R \sin (\theta), \\
& \dot{\theta}=D z+H \sin (\theta),
\end{aligned}
$$

where $x=(z, \theta) \in \mathbb{R}^{n}$ is the state, and $F \in \mathbb{R}^{k \times k}, R \in \mathbb{R}^{k \times q}$, $D \in \mathbb{R}^{q \times k}$ and $H \in \mathbb{R}^{q \times q}$ are constant matrices. Note that the system has an equilibrium at the origin. 
Let the symbol $\operatorname{diag}(b)$ denote the diagonal matrix with a vector $b \in \mathbb{R}_{+}^{q}$ on the main diagonal. Then impose the following restrictions on the dynamics of (12):

Assumption 3. There exist matrices $P=P^{\top} \in \mathbb{R}^{k \times k}, Q=$ $Q^{\top} \in \mathbb{R}^{k \times k}$ and a vector $m \in \mathbb{R}_{+}^{q}$ such that the following linear matrix inequalities are satisfied:

$$
\begin{gathered}
F^{\top} P+P F=-Q, P>0, Q>0, \\
2 P R=-D^{\top} \operatorname{diag}(m), \operatorname{diag}(m) H+H^{\top} \operatorname{diag}(m)<0 .
\end{gathered}
$$

Since Assumption 3 implies that the matrix $F$ is stable, it is straightforward to establish boundedness of $z \in \mathbb{R}^{k}$. The result below demonstrates how the approach derived in Corollary 2 can be used to also infer global boundedness of the variables $\theta \in \mathbb{R}^{q}$.

Proposition 3. Let Assumption 3 be satisfied for the system (12). Then for all $x_{0} \in \mathbb{R}^{n}$ the solutions $X\left(t, x_{0}\right)$ are bounded for all $t \geq 0$.

Proof. Under the made hypothesis let us consider the function

$$
V(x)=z^{\top} P z+\sum_{i=1}^{q} m_{i}\left(1-\cos \left(\theta_{i}\right)\right)-c,
$$

where $m_{i}$ is the $i^{\text {th }}$ entry of the vector $m$ and let us check the conditions of Corollary 2 for a Leonov function candidate $V(x)$. Since $1-\cos (\theta) \geq 0$, select $\alpha(s)=\lambda_{\min }(P) s^{2}$ (here $\lambda_{\min }(P)$ denotes the smallest eigenvalue of the matrix $P$ ), $\psi$ as any function from class $\mathcal{K}$ and $g=c$. Then the lower bound on $V(x)$ is verified for all $x \in \mathbb{R}^{n}$. Since $\cos (0)=1$ and it is a $2 \pi$-periodic function, the condition $\sup _{x \in \mathcal{U}} V(x) \leq$ 0 is satisfied for any $c \geq 0$. Select $\mathcal{W}=\{x=(z, \theta) \in$ $\left.\mathbb{R}^{n}:|\theta|_{\infty}=p\right\}$ for some $p \in(\pi, 2 \pi)$ and $0 \leq c<(1-$ $\cos (p)) \inf _{1 \leq i \leq q} m_{i}$. Then $\inf _{x \in \mathcal{W}} V(x)>0$, and all required in (3) properties hold.

It remains to check the derivative of the Leonov function $V(x)$ and the following upper estimate:

$$
\frac{\partial V(x)}{\partial x} f(x) \leq-\lambda V(x), \quad \forall x \in\left(\mathbb{R}^{n} \backslash \Omega_{0}\right) \cap\left(\mathcal{Z} \cup \Omega_{\varepsilon}\right),
$$

where $\Omega_{\varepsilon}=\left\{x \in \mathbb{R}^{n}: V(x) \leq \varepsilon\right\}$ and $\mathcal{Z}=\{x=(z, \theta) \in$ $\left.\mathbb{R}^{n}:|z| \geq \zeta\right\}$ for some $\lambda>0, \varepsilon>0$ and $\zeta>0$. Lengthy but straightforward calculations show that

$$
\begin{aligned}
\frac{\partial V(x)}{\partial x} f(x)+ & \lambda V(x) \leq-z^{\top}(Q-\lambda P) z-\sin ^{\top}(\theta) \mathcal{H} \sin (\theta) \\
& +\lambda\left[\sum_{i=1}^{q} m_{i}\left(1-\cos \left(\theta_{i}\right)\right)-c\right] \\
\leq & -z^{\top}(Q-\lambda P) z+\sum_{i=1}^{q} v_{i}\left(\theta_{i}\right)
\end{aligned}
$$

where

$$
\begin{gathered}
\mathcal{H}=-0.5\left(\operatorname{diag}(m) H+H^{\top} \operatorname{diag}(m)\right)>0, \\
v_{i}\left(\theta_{i}\right)=\lambda m_{i}\left(1-\cos \left(\theta_{i}\right)\right)-\lambda_{\min }(\mathcal{H}) \sin ^{2}\left(\theta_{i}\right)-\lambda q^{-1} c .
\end{gathered}
$$

Note that under Assumption 3 there is a value of $\lambda$ sufficiently small such that $Q>\lambda P$, then $\zeta>0$ exists as needed (the functions $v_{i}\left(\theta_{i}\right)$ are globally bounded). The property

$$
v_{i}\left(\theta_{i}\right)>0
$$

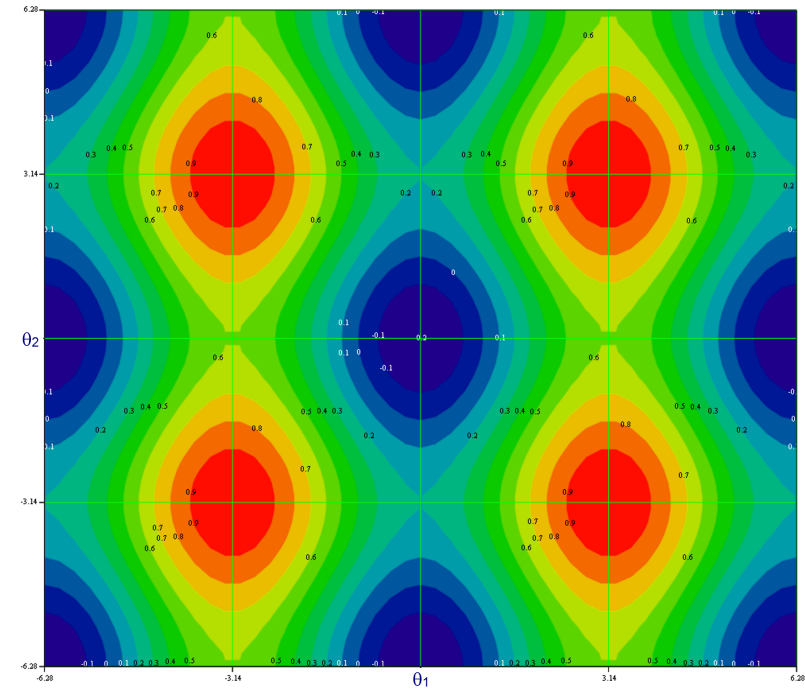

Figure 3. The levels of the function $V(0, \theta)$ for the system (12)

is valid for a small vicinity of the point $\theta_{i}=\pi$ (the negative term $-\lambda_{\min }(\mathcal{H}) \sin ^{2}\left(\theta_{i}\right)$ of (13) equals zero for $\theta_{i}=0$ or $\theta_{i}=\pi$, and their shifts on $2 \pi$, but the former is in $\Omega$ and only the latter point belongs to $\mathbb{R}^{n} \backslash \Omega$ by construction above). Furthermore, there also exists a sufficiently small $\lambda>0$ such that this vicinity has no intersection with $\Omega$. Indeed, $\lambda\left[m_{i}(1-\right.$ $\left.\left.\cos \left(\theta_{i}\right)\right)-q^{-1} c\right] \leq \lambda\left[2 m_{i}-q^{-1} c\right]$ for all $\theta_{i} \in[-\pi, \pi)$. In addition, since $\lambda_{\min }(\mathcal{H})>0$ by Assumption 3, then the inequality (13) can only be satisfied for $\theta_{i} \in\left(\pi-\rho_{i}, \pi+\rho_{i}\right)$ with $\rho_{i}=\arcsin \sqrt{\lambda \frac{2 m_{i}-q^{-1} c}{\lambda_{\min }(\mathcal{H})}}$, whose value can be selected as small as required by decreasing $\lambda$, i.e. $\varepsilon>0$ exists. The union of these restrictions means that the inequality (4) is not valid for $\pi-\max _{1 \leq i<q} \rho_{i} \leq|\theta|_{\infty} \leq \pi+\max _{1<i<q} \rho_{i}$ and $|z|<\zeta$ with some $\zeta>0$ (for a sufficiently small $\lambda$ ). However, all conditions of Corollary 2 are satisfied.

Example 3. Select $k=1, q=2$ and $F=-0.1, P=1$, $Q=0.2, R=-\left[\begin{array}{ll}0.2 & 0.05\end{array}\right], D=\left[\begin{array}{ll}1 & 0.5\end{array}\right]^{\top}, m=\left[\begin{array}{ll}0.4 & 0.2\end{array}\right]^{\top}$, $c=0.2$, and

$$
H=\left[\begin{array}{cc}
-1 & -0.25 \\
0.5 & -1
\end{array}\right]
$$

Then all conditions of Assumption 3 are satisfied for $\lambda=0.1$. The levels of the function $V(x)$ for $z=0$ and $\theta \in$ $[-2 \pi, 2 \pi]^{2}$ are presented in Fig. 3, the levels of the function $s(\theta)=\lambda\left[\sum_{i=1}^{q} m_{i}\left(1-\cos \left(\theta_{i}\right)\right)-c\right]-\sin ^{\top}(\theta) \mathcal{H} \sin (\theta)$ corresponding to verification of (13) are shown in Fig. 4, where comparing the zones of positivity for $V(0, \theta)$ and $s(\theta)$ it is possible to check that the inequality (4) is verified for $x \in\left(\mathbb{R}^{n} \backslash \Omega\right) \cap\left(\mathcal{Z} \cup \Omega_{\varepsilon}\right)$ with some $\varepsilon>0$. Illustrative simulation results for this system are given in Fig. 5. Clearly, the simulations confirm the efficiency of the proposed theory.

\section{CONCLUSIONS}

By extending ideas of [31], we have proposed a multivariable cell structure approach, which provides sufficient conditions to establish boundedness of trajectories of nonlinear 


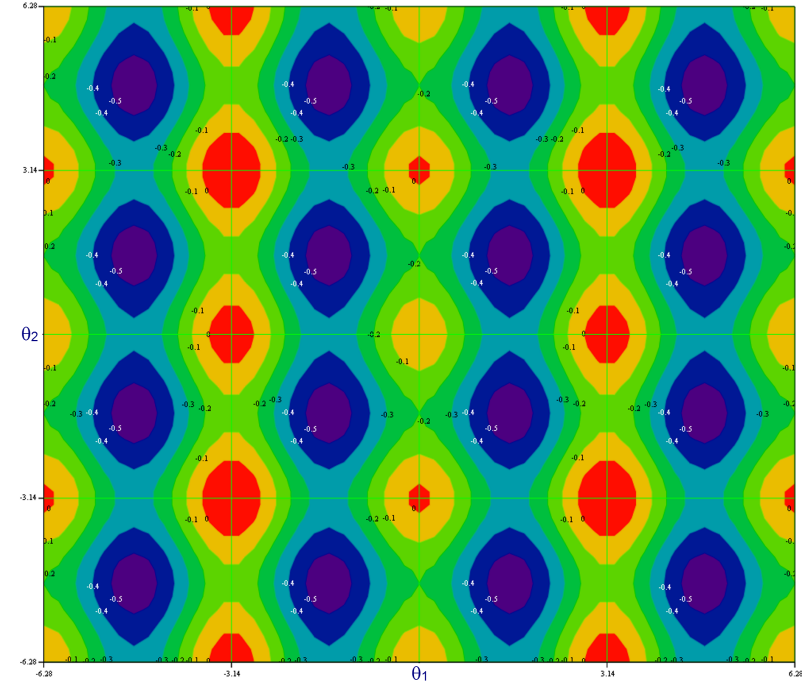

Figure 4. The levels of the function $s(\theta)$ for the system (12)

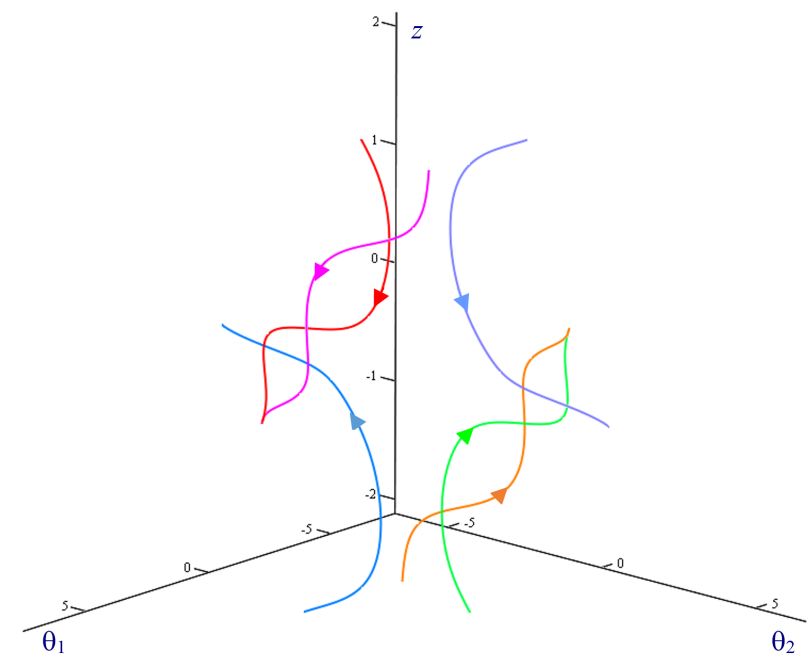

Figure 5. Simulation results for the system (12) for several arbitrarily chosen initial conditions

systems, the dynamics of which are periodic with respect to a part of the state vector. Key to the approach is the concept of a Leonov function. A Leonov function is in general signindefinite and not continuously differentiable on the manifold, on which the system dynamics can be projected. These characteristics represent significant relaxations compared to the usual requirements on a standard Lyapunov function [3]. We have shown that for a class of periodical dynamical systems the existence of a Leonov function implies boundedness of all trajectories. Furthermore, we have shown that under (mild) additional conditions a Leonov function is also an ISS-Leonov function (as defined in [18]) and thus its existence can also guarantee robustness with respect to exogenous disturbances. The proposed approach is illustrated by providing a global analysis of a nonlinear system. Application of the proposed theory to power systems and microgrids (in particular, to the swing equation) is currently under investigation.

\section{REFERENCES}

[1] D. Angeli. An almost global notion of input-to-state stability. IEEE Trans. Automatic Control, 49:866-874, 2004.

[2] D. Angeli and D. Efimov. On input-to-state stability with respect to decomposable invariant sets. In Proc. 52nd IEEE Conference on Decision and Control, pages 5897-5902, Florence, 2013.

[3] D. Angeli and D. Efimov. Characterizations of input-to-state stability for systems with multiple invariant sets. IEEE Trans. Automatic Control, 60(12):3242-3256, 2015.

[4] D. Angeli, J.E. Ferrell, and E.D. Sontag. Detection of multistability, bifurcations and hysteresis in a large class of biological positivefeedback systems. Proc. Natl. Acad. Sci. USA, 101:1822-1827, 2004.

[5] D. Angeli and L. Praly. Stability robustness in the presence of exponentially unstable isolated equilibria. IEEE Trans. Automatic Control, 56:1582-1592, 2011.

[6] D. Angeli and E.D. Sontag. Multi-stability in monotone input/output systems. Systems\&Control Lett., 51:185-202, 2004.

[7] Nikita Barabanov, Johannes Schiffer, Romeo Ortega, and Denis Efimov. Conditions for almost global attractivity of a synchronous generator connected to an infinite bus. IEEE Transactions on Automatic Control, 62(10):4905-4916, 2017.

[8] N.P. Bhatia and G.P. Szegö. Stability Theory of Dynamical Systems. Springer-Verlag, Berlin, 1970.

[9] M. Chaves, T. Eissing, and F. Allgöwer. Bistable biological systems: A characterization through local compact input-to-state stability. IEEE Trans. Automatic Control, 45:87-100, 2008.

[10] N.G. Chetaev. The Stability of Motion. Pergamon Press, New York, 1961. (English translation).

[11] Claudio De Persis and Nima Monshizadeh. Bregman storage functions for microgrid control. IEEE Transactions on Automatic Control, 63(1):53-68, 2018.

[12] F. Dörfler and F. Bullo. Synchronization and transient stability in power networks and non-uniform Kuramoto oscillators. SIAM Journal on Control and Optimization, 50(3):1616-1642, 2012.

[13] F. Dörfler and F. Bullo. Synchronization in complex networks of phase oscillators: A survey. Automatica, 50(6):1539-1564, 2014.

[14] Dawid Dudkowski, Sajad Jafari, Tomasz Kapitaniak, Nikolay V. Kuznetsov, Gennady A. Leonov, and Awadhesh Prasad. Hidden attractors in dynamical systems. Physics Reports, 637:1-50, 2016.

[15] D. Efimov. Global Lyapunov analysis of multi-stable nonlinear systems. SIAM Journal on Optimization and Control, 50(5):3132-3154, 2012.

[16] D. Efimov, W. Perruquetti, and M. Petreczky. On necessary conditions of instability and design of destabilizing controls. In Proc. 53rd IEEE Conference on Decision and Control, pages 3915-3917, Los Angeles, US, 2014.

[17] D. Efimov and J. Schiffer. A new criterion for boundedness of solutions for a class of periodic systems. In Proc. 17th European Control Conference (ECC), Limassol, 2018.

[18] D. Efimov, J. Schiffer, N. Barabanov, and R. Ortega. A relaxed characterization of ISS for periodic systems with multiple invariant sets. European Journal of Control, 37(9):1-7, 2017.

[19] D. Efimov, J. Schiffer, and R. Ortega. Robustness of delayed multistable systems with application to droop-controlled inverter-based microgrids. International Journal of Control, 89(5):909-918, 2016.

[20] D.V. Efimov and A.L. Fradkov. Oscillatority of nonlinear systems with static feedback. SIAM Journal on Optimization and Control, 48(2):618640, 2009.

[21] F. Forni and R. Sepulchre. Differential analysis of nonlinear systems: Revisiting the pendulum example. In Proc. 53rd IEEE Conference on Decision and Control, pages 3848-3859, Los Angeles, US, 2014.

[22] F. Forni and R. Sepulchre. A differential Lyapunov framework for contraction analysis. IEEE Transactions on Automatic Control, 59(3):614628, 2014.

[23] Fulvio Forni. Differential positivity on compact sets. In Decision and Control (CDC), 2015 IEEE 54th Annual Conference on, pages 63556360. IEEE, 2015.

[24] A.Kh. Gelig, G.A. Leonov, and V.A. Yakubovich. Stability of nonlinear systems with non unique equilibrium. Nauka, Moscow, 1978. [in Russian].

[25] J. Guckenheimer and P.J. Holmes. Nonlinear Oscillations, Dynamical Systems, and Bifurcations of Vector Fields, volume 42 of Applied Mathematical Sciences. Springer-Verlag, New York, 1983.

[26] W. Hahn. Stability of Motion. Springer-Werlag, 1967.

[27] H.K. Khalil. Nonlinear Systems. Macmillan, 1992.

[28] V. Lakshmikantham and X.Z. Liu. Stability analysis in terms of two measures. World Scientific, 1993. 
[29] G. A. Leonov, N. V. Kuznetsov, M. V. Yuldashev, and R. V. Yuldashev. Analytical method for computation of phase-detector characteristic. IEEE Transactions on Circuits and Systems II: Express Briefs, 59(10):633-637, 2012.

[30] G. A. Leonov, N. V. Kuznetsov, M. V. Yuldashev, and R. V. Yuldashev. Hold-in, pull-in, and lock-in ranges of pll circuits: Rigorous mathematical definitions and limitations of classical theory. IEEE Transactions on Circuits and Systems I: Regular Papers, 62(10):2454-2464, 2015.

[31] G.A. Leonov. On the boundedness of the trajectories of phase systems. Siberian Mathematical Journal, 15(3):491-495, 1974.

[32] W. Lohmiller and J.S. Slotine. On contraction analysis of nonlinear systems. Automatica, 34(6):683-696, 1998.

[33] A.M. Lyapunov. The general problem of the stability of motion. Taylor \& Francis, London, 1992.

[34] Z. Nitecki and M. Shub. Filtrations, decompositions, and explosions. American Journal of Mathematics, 97(4):1029-1047, 1975.

[35] E.J. Noldus. New direct Lyapunov-type method for studying synchronization problems. Automatica, 13:139-151, 1977.

[36] R. Ortega, M. Galaz, A. Astolfi, Y. Sun, and T. Shen. Transient stabilization of multimachine power systems with nontrivial transfer conductances. IEEE Trans. Automatic Control, 50(1):60 - 75, jan. 2005

[37] Alexey Pavlov, Alexander Pogromsky, Nathan van de Wouw, and Henk Nijmeijer. Convergent dynamics, a tribute to boris pavlovich demidovich. Systems \& Control Letters, 52(3-4):257-261, 2004.

[38] A. Rantzer. A dual to Lyapunov's stability theorem. Systems\&Control Lett., 42:161-168, 2001.

[39] V.V. Rumyantsev and A.S. Oziraner. Stability and stabilization of motion with respect to part of variables. Nauka, Moscow, 1987. [in Russian].

[40] J. Schiffer and F. Dörfler. On stability of a distributed averaging PI frequency and active power controlled differential-algebraic power system model. In Proc. European Control Conference, pages 14871492, Aalborg, DK, 2016.

[41] J. Schiffer, D. Efimov, and R. Ortega. Almost global synchronization in multi-machine power systems. In Proc. 57th IEEE Conference on Decision and Control (CDC), Fontainebleau, FL, 2018.

[42] J. Schiffer, R. Ortega, A. Astolfi, J. Raisch, and T. Sezi. Conditions for stability of droop-controlled inverter-based microgrids. Automatica, 50(10):2457-2469, 2015.

[43] Johannes Schiffer, Denis Efimov, Romeo Ortega, and Nikita Barabanov. An input-to-state stability approach to verify almost global stability of a synchronous-machine-infinite-bus system. Phil. Trans. R. Soc. A, 375(2100):20160304, 2017

[44] G.-B. Stan and R. Sepulchre. Analysis of interconnected oscillators by dissipativity theory. IEEE Trans. Automatic Control, 52:256-270, 2007.

[45] Yang Tang, Feng Qian, Huijun Gao, and Jürgen Kurths. Synchronization in complex networks and its application-a survey of recent advances and challenges. Annual Reviews in Control, 38(2):184-198, 2014.

[46] Sebastian Trip, Mathias Bürger, and Claudio De Persis. An internal model approach to (optimal) frequency regulation in power grids with time-varying voltages. Automatica, 64:240-253, 2016.

[47] A.J. van der Schaft. L2-Gain and Passivity Techniques in Nonlinear Control. Springer, 2000.

[48] V.A. Yakubovich, G.A. Leonov, and A.Kh. Gelig. Stability of stationary sets in control systems with discontinuous nonlinearities, volume 14 of Series on stability, vibration, and control of systems. World Scientific, 2004.

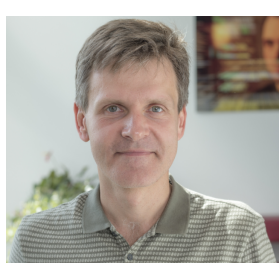

Denis Efimov (SM'11) received the Ph.D. degree in Automatic Control from the Saint-Petersburg State Electrical Engineering University (Russia) in 2001, and the Dr.Sc. degree in Automatic control in 2006 from Institute for Problems of Mechanical Engineering RAS (Saint-Petersburg, Russia). From 2000 to 2009 he was research fellow of the Institute for Problems of Mechanical Engineering RAS, Control of Complex Systems Laboratory. From 2006 to 2011 he was working in the LSS (Supelec, France), the Montefiore Institute (University of Liege, Belgium) and the Automatic control group at IMS lab (University of Bordeaux I, France). Since 2011 he joined Inria. He is a member of several IFAC TCs and a Senior member of IEEE. His main research interests include oscillation analysis, observation, control and nonlinear system stability.

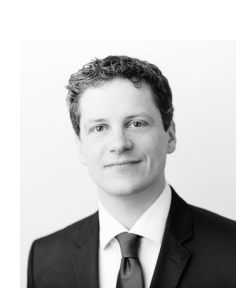

Johannes Schiffer received the Diploma degree in engineering cybernetics from the University of Stuttgart, Stuttgart, Germany, in 2009 and the Ph.D. degree (Dr.-Ing.) in electrical engineering from Technische Universität (TU) Berlin, Berlin, Germany, in 2015. He currently holds the chair of Control Systems and Network Control Technology at Brandenburgische Technische Universität CottbusSenftenberg, Cottbus, Germany. Prior to that, he has held appointments as Lecturer (Assistant Professor) at the School of Electronic and Electrical Engineering, University of Leeds, Leeds, U.K. and as Research Associate in the Control Systems Group and at the Chair of Sustainable Electric Networks and Sources of Energy both at TU Berlin. In 2017 he and his co-workers received the Automatica Paper Prize over the years 2014-2016. His current research interests include distributed control and analysis of complex networks with application to microgrids and power systems. 